Subscriber access provided by EPFL | Scientific Information and Libraries

Article

\title{
Polymer-Grafted Mesoporous Silica Nanoparticles as Ultrasound-Responsive Drug Carriers
}

Juan L Paris, M. Victoria Cabañas, Miguel Manzano, and María Vallet-Regí

ACS Nano, Just Accepted Manuscript • DOI: 10.1021/acsnano.5b04378 • Publication Date (Web): 11 Oct 2015

Downloaded from http://pubs.acs.org on October 11, 2015

\section{Just Accepted}

"Just Accepted" manuscripts have been peer-reviewed and accepted for publication. They are posted online prior to technical editing, formatting for publication and author proofing. The American Chemical Society provides "Just Accepted" as a free service to the research community to expedite the dissemination of scientific material as soon as possible after acceptance. "Just Accepted" manuscripts appear in full in PDF format accompanied by an HTML abstract. "Just Accepted" manuscripts have been fully peer reviewed, but should not be considered the official version of record. They are accessible to all readers and citable by the Digital Object Identifier (DOI®). "Just Accepted" is an optional service offered to authors. Therefore, the "Just Accepted" Web site may not include all articles that will be published in the journal. After a manuscript is technically edited and formatted, it will be removed from the "Just Accepted" Web site and published as an ASAP article. Note that technical editing may introduce minor changes to the manuscript text and/or graphics which could affect content, and all legal disclaimers and ethical guidelines that apply to the journal pertain. ACS cannot be held responsible for errors or consequences arising from the use of information contained in these "Just Accepted" manuscripts. 


\title{
Polymer-Grafted Mesoporous Silica
}

\section{Nanoparticles as Ultrasound-Responsive Drug}

\section{Carriers}

\author{
Juan L. Paris,,${ }^{\&,+}$ M. Victoria Cabañas, ${ }^{\&}$ Miguel Manzano ${ }^{\&,+}$ and María Vallet-Regi ${ }^{\ell,+}, *$. \\ ${ }^{\&}$ Dpto. Química Inorgánica y Bioinorgánica, Facultad de Farmacia, UCM, Instituto de \\ Investigación Sanitaria Hospital 12 de Octubre i+12, Madrid, Spain. ${ }^{+}$Centro de Investigación \\ Biomédica en Red de Bioingeniería, Biomateriales y Nanomedicina (CIBER-BBN), Spain.
}

\begin{abstract}
A new ultrasounds-responsive system based on mesoporous silica nanoparticles was developed for biomedical applications, grafting a co-polymer on their surface that acts as gatekeeper of the pores. The nanoparticles can be loaded with a cargo at low temperature $\left(4^{\circ} \mathrm{C}\right)$, taking advantage of the open conformation that the polymer presents under these conditions. Then, at $37^{\circ} \mathrm{C}$ the copolymer collapses closing the pore entrances and allowing the nanoparticles to carry the drugs at physiological temperature without premature release, which is of great importance when dealing with cytotoxic drugs in cancer treatments. Upon ultrasounds irradiation, the sensitive polymer changes its hydrophobicity and, therefore, its conformation towards coil-like opening the gates and releasing the cargo. These hybrid nanoparticles have been shown to be non-cytotoxic and can be internalized into $\mathrm{LNCaP}$ cells retaining their ultrasound responsive capability in the cytoplasm of the cells. Moreover, doxorubicin-loaded
\end{abstract}


hybrid MSNs were incubated with LNCaP cells to show their capacity to induce cell death only when the nanoparticles had been exposed to ultrasound. This work demonstrates that our hybridMSNs can be triggered by remote stimuli, which is of capital importance for future applications in drug delivery and cancer therapy.

KEYWORDS: Mesoporous silica; ultrasound; stimuli-responsive; drug delivery; nanomedicine

Nanotechnology has fully transformed the area of drug delivery thanks to the use of nanocarriers for therapeutics. The reason behind that revolution is the potential for addressing some of the most significant restrictions of conventional medicine, such as poor drug solubility, inadequate pharmacokinetics and diverse side effects. Nanoparticles employed as drug delivery systems have displayed important benefits such as enhanced accumulation of drug molecules at diseased tissues and cells, contributing to the reduction of the systemic toxicity. ${ }^{1-3}$

Among all nanoparticles employed in biomedicine, mesoporous silica nanoparticles (MSNs) are very attractive nanocarriers for diagnostic and therapeutic applications. ${ }^{4-7}$ MSNs present unique material properties, such as mechanical and chemical stability, good biocompatibility, and high loading capacity. In fact, the excellent textural properties of mesoporous silica, such as high surface area, high pore volume and tunable pore sizes, guarantee a great loading capacity and efficient encapsulation of an immense variety of cargo molecules. ${ }^{8-10}$ Additionally, their chemical composition assures the efficient attachment of organic surface functionalities such as gate keepers to avoid premature release of the cargo, or targeting ligands to favor selective localization of MSNs towards diseased tissues.

However, it is very difficult to actually control the release of the drug from the nanocarriers, which normally relays on the biodegradation of the nanoparticles in vivo. One alternative that has been developed in the last few years is the concept of stimuli-responsive drug delivery systems, 
which allow for tailored release profiles with spatial, temporal and dosage control. ${ }^{7,10,11}$ Those stimuli-responsive systems consist on triggering the release of the therapeutic drug at the diseased site through the use diverse entities that are sensitive to exogenous stimuli (i.e. temperature, light, magnetic fields, electric fields and ultrasounds) or endogenous stimuli (i.e., changes in $\mathrm{pH}$, redox potential, or the concentrations of enzymes or specific analytes). ${ }^{12-15}$

Among feasible external stimuli, ultrasounds (US) represent a unique and exciting method for achieving spatiotemporal control of the drug release at the desired site. ${ }^{16}$ Additionally, US are attractive because of their non-invasiveness, the absence of ionizing radiations, the cost effectiveness and the easy regulation of tissue penetration depth by tuning the frequency, cycles and exposure time. In fact, high-frequency ultrasound can penetrate deep into the body with focused beams, which allows local therapy avoiding adverse side effects to healthy tissues. Moreover, ultrasound stimulus itself presents interesting properties for nanomedicine, as it has been shown to enhance nanoparticle extravasation through blood capillaries, increase cell membrane permeation and even induce an immune response against tumors. ${ }^{17,18}$

Ultrasound waves can induce thermal and/or mechanical effects that could trigger the release of the drug from a collection of nanocarriers, such as liposomes, micelles, microbubbles, etc. ${ }^{16}$ Moreover, advances in sonochemistry have shown that ultrasound-induced chemical reactions can differ from those carried out by bulk heating, implying mechanical or thermal effects at the nanoscale. $^{19}$ US irradiation can also cleave certain chemical bonds, the so called mechanophores. ${ }^{20,21}$ This strategy can be exploited in the design of responsive nanoparticles with ultrasound-labile moieties. In this context, 2-Tetrahydropyranyl methacrylate (THPMA) is a hydrophobic monomer bearing a labile acetal group that can be cleaved by ultrasound to yield hydrophilic methacrylic acid (MAA). ${ }^{20,22}$ This strategy, phase transformation from hydrophobic 
to hydrophilic by US irradiation, could be used to develop a polymeric gatekeeper on nanoparticles to avoid premature release of different cargos.

Regarding the cargo of different molecules in MSNs, nanogates based on thermosensitive polymers that exhibit a lower critical solution temperature (LCST), are a versatile option for this purpose. These polymers are soluble in aqueous medium at low temperature, but upon heating, when the system reaches the LCST, the polymer changes to a hydrophobic state, collapsing and giving rise to a suspension in the same aqueous medium. This means that by changing the temperature of the medium, the use of thermosensitive polymers with a LCST allows loading a drug in MSNs after anchoring the polymer nanogates to their surface. ${ }^{12,13,23,24}$ This constitutes a great advantage over most stimuli-responsive materials, that must be loaded with the cargo prior to the nanogate grafting. ${ }^{25}$

The combination of a thermoresponsive polymer, such as poly(2-(2-methoxyethoxy)ethyl methacrylate), $\mathrm{p}\left(\mathrm{MEO}_{2} \mathrm{MA}\right),{ }^{26}$ with an ultrasound responsive monomer (THPMA) would result in a dual-responsive copolymer: the thermal response allows us to load and retain the cargo, and the US sensitivity to induce its release at physiological temperature. The US-responsive character empowers the thermoresponsive polymer with the particular property of modulating its phase state by US irradiation at a chosen temperature. Besides, both components of the designed copolymer $\left(\mathrm{MEO}_{2} \mathrm{MA}\right.$ and THPMA) have been shown to present good biocompatibility and interesting properties for biomedical application. ${ }^{27,28}$

The mentioned advantages of US irradiation as extern stimulus motived us to explore a novel smart drug delivery system. In this work, we have combined the possibilities of US stimulus together with the great capabilities of MSNs to design an US stimulus-responsive drug delivery system. Despite the great potential of this strategy, the use of US in mesoporous silica remains 
largely unexplored. ${ }^{29,30}$ Basically, we have developed a nanocarrier based on MSNs with nanogates that allows the encapsulation and transportation of drugs with no premature release to specific locations in the body where the cargo can be released upon externally applied ultrasounds (Scheme 1). To achieve that, we have functionalized MSNs with a copolymer able to open and close the gates of the carrier pores through a nanogate. Copolymer $\mathrm{p}\left(\mathrm{MEO}_{2} \mathrm{MA}\right)$-coTHPMA bearing US-cleavable hydrophobic tetrahydropyranyl moieties, presents a LCST below physiological temperature. At $4^{\circ} \mathrm{C}$, the polymer is in its coil-like conformation, allowing the cargo to be loaded in the mesopores. When the temperature is increased to physiological temperature, the copolymer changes to a collapsed state (insoluble) and the nanogates are closed retaining the cargo into the pores. Upon US irradiation, the hydrophobic tetrahydropyranyl moieties are cleaved, leading to an increase of the hydrophilicity of the polymer and, therefore, an increase of the LCST over physiological temperature. This induces a change in conformation of the polymer to coil-like, opening the gates of the mesopores of the MSNs and allowing the entrapped cargo to be released (Scheme 1).
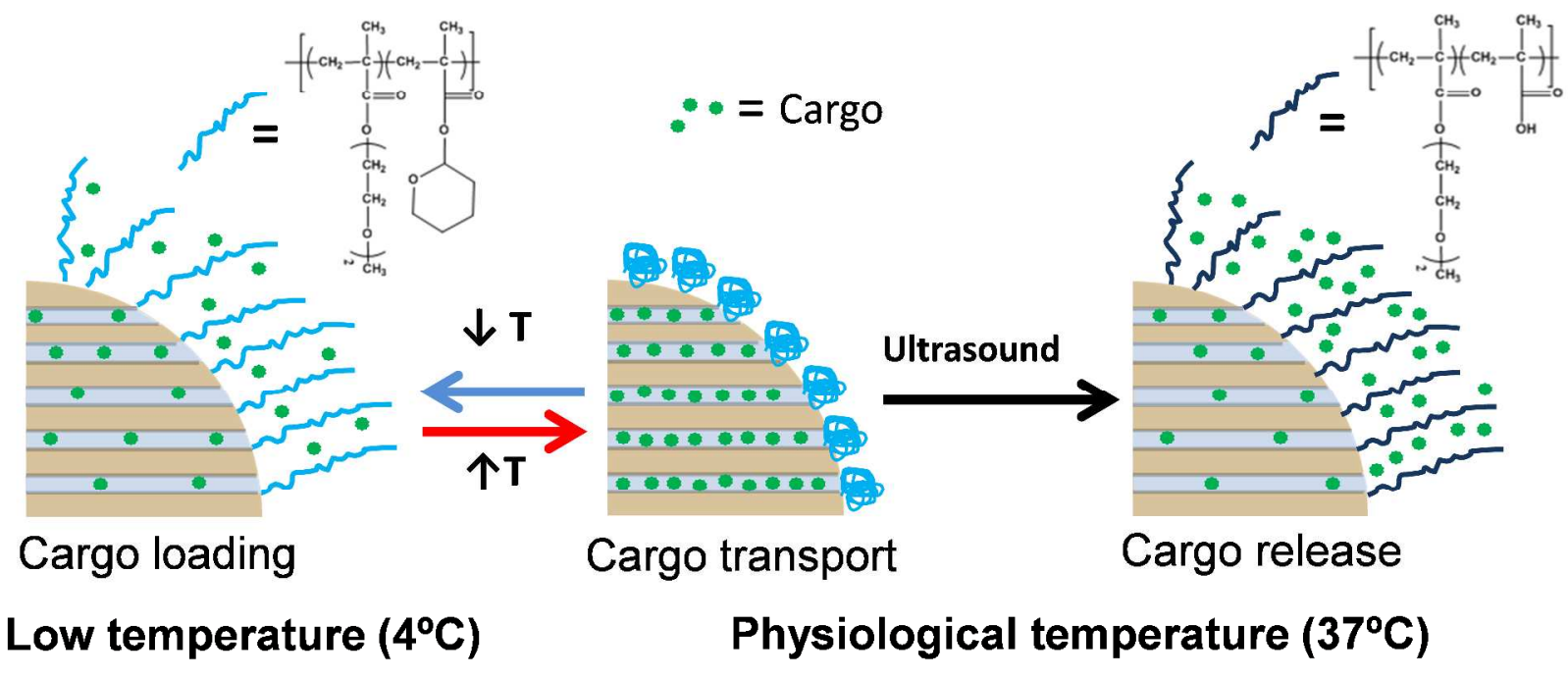

Cargo transport

Cargo release

Physiological temperature $\left(37^{\circ} \mathrm{C}\right)$

Scheme 1. Schematic illustration of the behavior in aqueous medium of dual responsive release system. 
In this work, we propose and demonstrate a new approach to stimuli-responsive systems by using hybrid mesoporous silica nanoparticles that allow encapsulation of small molecules of any type that would be released only upon external US irradiation.

\section{RESULTS AND DISCUSSION}

Hybrid-MSN nanoparticles. Mesoporous silica nanoparticles, MSNs, with a MCM-41 type structure, diameter of $c a .200 \mathrm{~nm}$ and $2 \mathrm{~nm}$ mesopores were synthesized by a modification of the well-known Stöber method. The external surface of the MSNs was coated with a copolymer able to open or close the pore entrances under certain stimuli. Thus, preparation and optimization of the copolymer properties was the first task to be completed.

The dual temperature-ultrasound-responsive random copolymer poly(2-(2methoxyethoxy) ethylmethacrylate-co-2-tetrahydropyranyl methacrylate, $\mathrm{p}\left(\mathrm{MEO}_{2} \mathrm{MA}\right.$-co-THPMA), was synthesized by free radical polymerization from 2-(2-methoxyethoxy) ethylmethacrylate, $\mathrm{MEO}_{2} \mathrm{MA}$ (temperature-responsive monomer) and 2-tetrahydropyranyl methacrylate, THPMA (ultrasound-responsive monomer). ${ }^{26}$ Different ratios of $\mathrm{MEO}_{2} \mathrm{MA}$ and THPMA monomers were evaluated to endow the copolymer with LCST lower (LCST1) and higher (LCST2) than $37^{\circ} \mathrm{C}$ before and after ultrasound irradiation, respectively (table S1, supporting information). Optimal results were achieved for a $\mathrm{MEO}_{2} \mathrm{MA}$ :THPMA ratio of $c a$. 90:10 (composition estimated by ${ }^{1} \mathrm{H}-$ NMR) synthesized from a 87.5:12.5 molar ratio. Lower content in US-responsive monomer lead to LCST2 lower than $37^{\circ} \mathrm{C}$, after 10 min of US irradiation. In fact, the total absence of THPMA in the polymer backbone resulted into a lack of responsiveness upon US irradiation: the LCST and ${ }^{1} \mathrm{H}-\mathrm{NMR}$ spectrum of the polymer were not modified after US exposure (Figure S1). On the other hand, for higher THPMA contents, the copolymer was insoluble in aqueous solution even 
at low temperature, which means that the copolymer was always in a collapsed state closing the gates of the pores. Consequently, it was not possible to load the cargo in this medium even at $4^{\circ} \mathrm{C}$. Therefore, 90:10 ( $\mathrm{MEO}_{2} \mathrm{MA}$ :THPMA ratio) was selected for the forthcoming experiments.

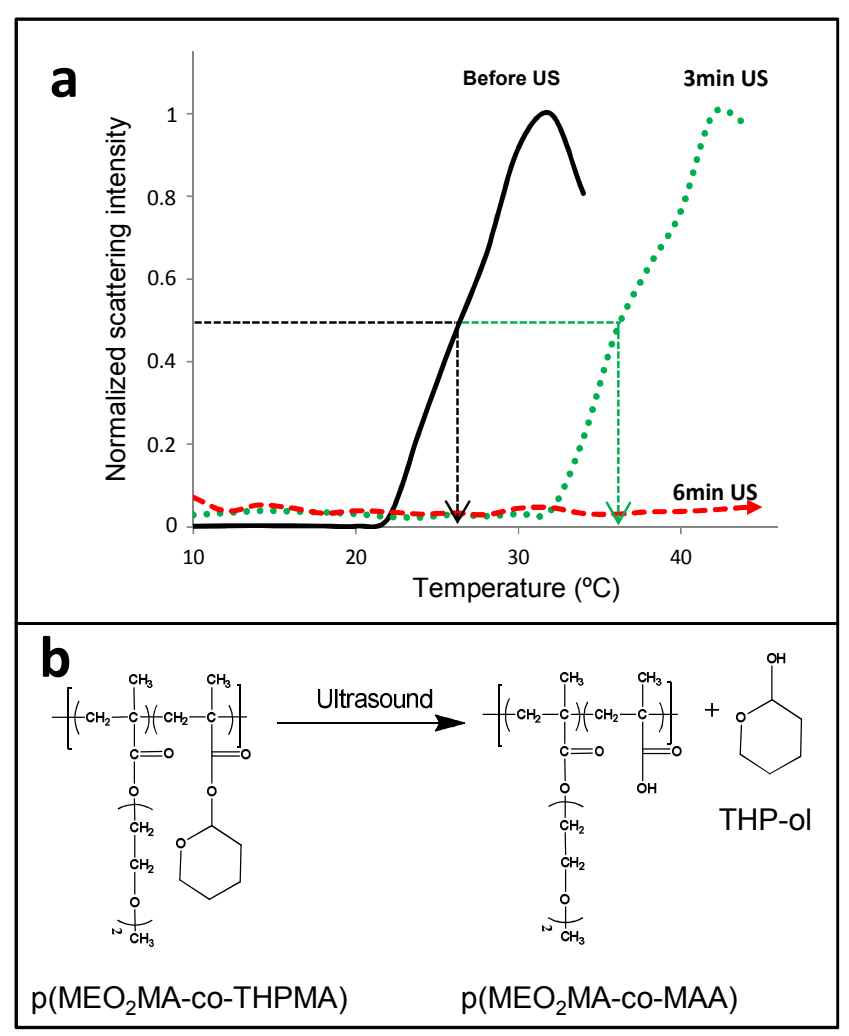

Figure 1. (a) Lower Critical Solution Temperature (LCST) of $\mathrm{p}\left(90 \mathrm{MEO}_{2} \mathrm{MA}-10 \mathrm{THPMA}\right)$ after different periods of time of ultrasound irradiation (1.3 MHz and $100 \mathrm{~W}$ ) (b) Hydrolysis of dualresponsive $\mathrm{p}\left(\mathrm{MEO}_{2} \mathrm{MA}-\mathrm{THPMA}\right)$ into $\mathrm{p}\left(\mathrm{MEO}_{2} \mathrm{MA}-\mathrm{MAA}\right)$ and THP-ol (tetrahydropyranol) by ultrasound.

The variation of the LSCT of this copolymer as a function of US irradiation time is collected in Figure 1a (the frequency and power of the US beam were fixed at $1.3 \mathrm{MHz}$ and $100 \mathrm{~W}$, respectively). As it can be observed, applied US time had a terrific influence on shifting the LCST of the polymer: the LCST1 of the as-synthesised copolymer was $26^{\circ} \mathrm{C}$, after 3 minutes of US irradiation it shifted to $36^{\circ} \mathrm{C}$, and after 6 minutes of US beam the LCST2 went higher than $45^{\circ} \mathrm{C}$. The LCST increase can be attributed to the cleavage of the sensitive part of the copolymer, THPMA, through the tetrahydropyranyl group (Figure 1b). ${ }^{20,22}$ This cleavage leads to a change 
in the composition and hydrophobicity of the copolymer and, therefore, to a LCST shift to temperatures above $37^{\circ} \mathrm{C}$ modifying the polymer state towards an extended conformation. The ${ }^{1} \mathrm{H}$ NMR spectrum of the copolymer after US irradiation (Figure S2) confirms the disappearance of the THP moiety. To ensure that THPMA cleavage was not due to bulk heating caused by the US application, a solution of the selected copolymer was heated to $80^{\circ} \mathrm{C}$ for $30 \mathrm{~min}$. No modification in the LCST or the ${ }^{1} \mathrm{H}$ NMR spectrum could be found (Figure S2). Also, the THP group was removed by acid catalysis with $p$-toluenesulfonic acid in methanol for $5 \mathrm{~h}$, and the changes in the ${ }^{1} \mathrm{H}$ NMR spectrum were the same as those found when the monomer THPMA was cleaved by US application (Figure S2). These experiments prove that the changes observed are unequivocally due to US irradiation. Then, the ultrasound-induced rise in the LCST makes the tested copolymer and ratio suitable for the desired application.

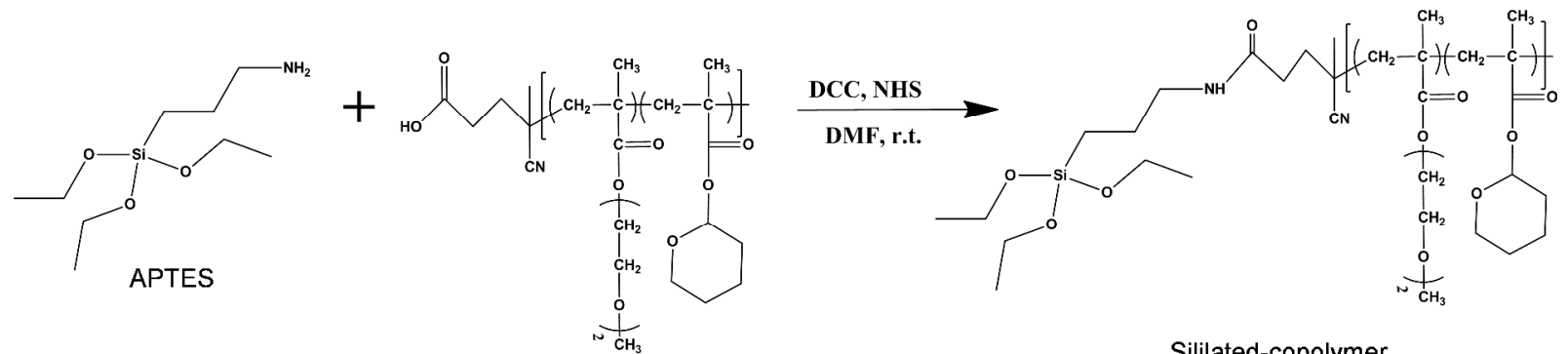

$\mathrm{p}\left(\mathrm{MEO}_{2} \mathrm{MA}-\mathrm{CO}-\mathrm{THPMA}\right)$

Sililated-copolymer

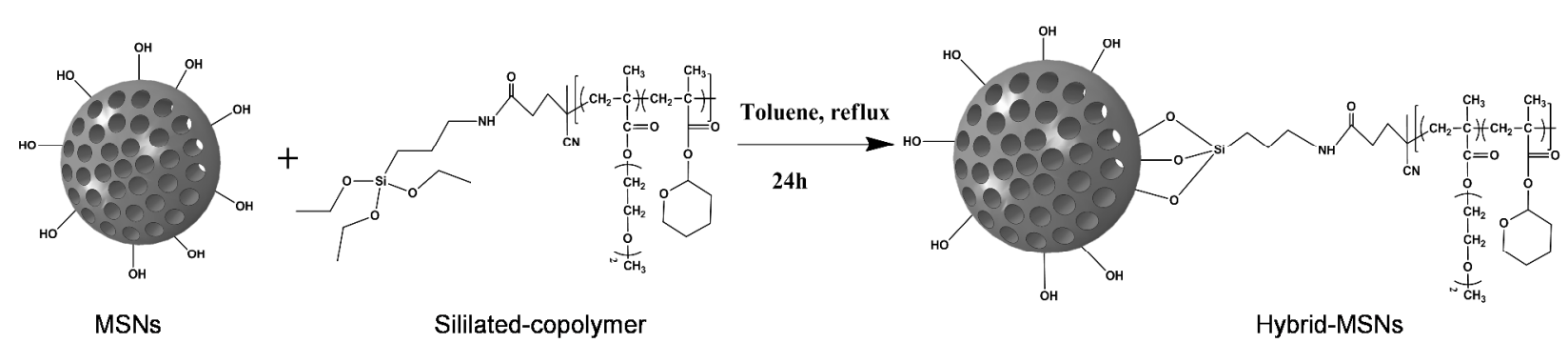

Scheme 2. Preparation of $\mathrm{p}\left(\mathrm{MEO}_{2} \mathrm{MA}-\mathrm{co}-\mathrm{THPMA}\right)-\mathrm{SiO}_{2}$ nanoparticles (Hybrid-MSNs):APTES conjugation to $\mathrm{p}\left(\mathrm{MEO}_{2} \mathrm{MA}-\mathrm{co}-\mathrm{THPMA}\right)$ (top) and grafting of the sililated-copolymer to mesoporous $\mathrm{SiO}_{2}$ nanoparticles (bottom).

Once both components of our system, nanocarrier and polymeric nanogates, were produced and optimized, the next step was joining them together. This procedure, described in Scheme 2, 
was based on functionalising the copolymer $\left(\mathrm{MEO}_{2} \mathrm{MA}-\mathrm{co}-\mathrm{THPMA}\right)$ with an alkoxysilane (3aminopropyl triethoxysilane) through DCC-NHS chemistry to form a sililated polymer, to then graft it to the silica nanoparticles surface through silanol chemistry. The ratio of polymer:nanoparticles together with the coupling reaction conditions were explored to obtain the best possible responsive system. Basically, the amount of grafted copolymer had to be optimized to effectively block the pore entrances of the nanoparticles to avoid any possible premature release. To evaluate that, the already functionalized nanoparticles with different amounts of polymer, hybrid-MSNs from now on, were loaded with fluorescein at $4^{\circ} \mathrm{C}$ (extended polymer conformation and open pore entrances) and then temperature was increased up to $37^{\circ} \mathrm{C}$, so the polymer collapsed closing the pore entrances with the dye molecules adsorbed into the mesopores. Then the percentage of dye released in $\mathrm{PBS}$ at $37^{\circ} \mathrm{C}$ in all the evaluated compositions (Table S2) was measured with a fluorimeter after 24 hours of incubation. When the sililated polymer was added in one step to the nanoparticles suspension in a $4: 1$ ratio (polymer:nanoparticles), the maximum amount of organic matter present in the inorganic nanocarriers measured by thermogravimetric analysis was $c a .21 \%$. However, the amount of fluorescein released after $24 \mathrm{~h}$ at $37^{\circ} \mathrm{C}$ was $c a .56 \%$, which means that half of the cargo was being prematurely released without any triggering event, so the copolymer chains were not perfectly closing the gates of the pores. Although increasing the polymer amount might seem as the straightforward solution for properly blocking the pore entrances, we found that $6: 1$ ratio resulted into even lower functionalization efficiency (ca. $15 \%$ of organic matter). We hypothesized that high concentration of sililated polymers into the reaction environment could lead to self-condensation rather than condensation with the nanoparticles surface. Consequently, we tested the same ratio, $6: 1$, but adding the sililated polymer in 3 steps rather than in 1 step, so 
the polymer concentration was kept low at all times and, therefore, avoiding self-condensation. That approach produced good enough results with ca. $30 \%$ of organic matter and only $26 \%$ of fluorescein released at $37^{\circ} \mathrm{C}$ after 24 hours.

The successful grafting of copolymer to mesoporous silica nanoparticles to form hybridMSNs was confirmed by different characterization techniques (see details in Supporting Information, Figure S3). In this sense, the presence of the typical vibrations bands of silica (between 490 and $1090 \mathrm{~cm}^{-1}$ ) together with those bands from the polymer (between 1400 and $1800 \mathrm{~cm}^{-1}$ ) in the FTIR spectra confirmed the functionalization process (Figure S3a). The lowangle XRD pattern of MSNs shows the characteristic ordered MCM-41 type mesostructured with 2D hexagonal (p6m) symmetry (Figure S3b). Similar maxima were observed in hybrid-MSNs, which means that the ordered mesostructured of the particles survived the functionalization process. MSNs showed the characteristic zeta potential of $-21 \mathrm{mV}$ at $\mathrm{pH}=7$, which was shifted to -31.6 $\mathrm{mV}$ after copolymer grafting. Thermogravimetry experiments also confirmed the presence of organic matter in hybrid-MSNs, as expected (Figure S3c). Nitrogen adsorption experiments confirmed the size of the mesopores (ca. $2.5 \mathrm{~nm}$ ) and also confirmed the successful grafting of the polymer chains thanks to the reduction of the surface area, from $934 \mathrm{~m}^{2} / \mathrm{g}$ in MSNs to 171 $\mathrm{m}^{2} / \mathrm{g}$ in hybrid-MSNs (Figure S3d). These data suggest that the external surface of the particles was covered with the copolymer blocking the mesopores entrances.

The morphology and size of MSNs and hybrid-MSNs are very similar, as can be seen in Figure 2. The spherical morphology of MSNs remained after the functionalization process as it can be observed in the SEM micrographs. The mean size was determined by Dynamic Light Scattering (DLS), and found to be $220 \mathrm{~nm}$ (MSNs) and $252 \mathrm{~nm}$ (hybrid-MSNs). In both cases, well-ordered mesoporous silica particles can be appreciated in the corresponding TEM 
micrographs. Table 1 resumes some of the most important nanoparticle characteristics before and after functionalization with the US-sensitive copolymer.

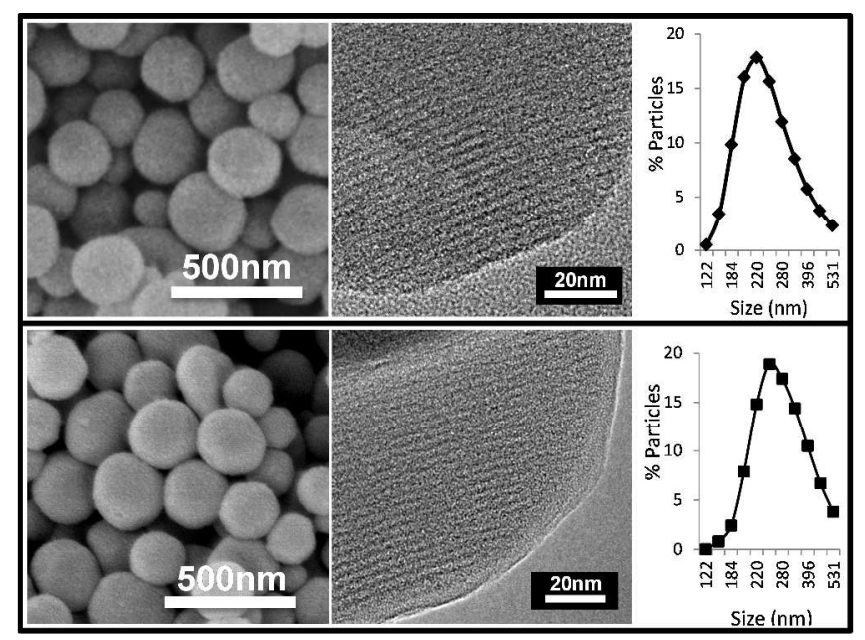

Figure 2. From left to right: SEM images, TEM micrographs and size distribution (measured by DLS) of MSNs (top) and hybrid-MSNs (bottom).

Table 1. Main features corresponding to MSNs and hybrid-MSNs.

\begin{tabular}{ccc}
\hline SAMPLE & MSNs & Hybrid-MSNs \\
\hline Z Potential & $-21.1 \mathrm{mV}$ & $-31.6 \mathrm{mV}$ \\
BET Surface Area & $935 \mathrm{~m}^{2} / \mathrm{g}$ & $171 \mathrm{~m}^{2} / \mathrm{g}$ \\
Size Mean (DLS) & $220 \mathrm{~nm}$ & $252 \mathrm{~nm}$ \\
Organic matter (TGA) & $5.1 \%$ & $30.5 \%$ \\
\hline
\end{tabular}

Finally and since these nanoparticles will be used in a biological application, their degradation in PBS solution over time was evaluated (Figure S4). After 8 days under physiological conditions, the mesostructure and morphology of the hybrid-MSNs remained almost unaffected, while MSNs started to degrade after 5 days of experiment. In this sense, hybrid-MSNs showed slower degradation rate than naked MSNs. This observation is in agreement with other results reported in the literature. ${ }^{31}$ 
In vial cargo release experiments. As mentioned above, one of the great advantages of thermoresponsive systems like the one here proposed relays on the loading process. Conventionally, functionalized MSNs must adsorb the cargo into the mesopores simultaneously to the grafting process, which leads to poor loading and functionalization yields and efficiency. However, our system allows opening the nanogates at $4^{\circ} \mathrm{C}$ after the functionalization process has been completed, loading the cargo, and then, closing the pore entrances just increasing the temperature to $37^{\circ} \mathrm{C}$.

Fluorescein was used as a model molecule to evaluate the ultrasound-responsiveness of the copolymer-grafted MSNs. Hybrid-MSNs were dispersed into a solution of $20 \mathrm{mg} / \mathrm{mL}$ of fluorescein dissolved in PBS at $4^{\circ} \mathrm{C}$ for $24 \mathrm{~h}$. This temperature is lower than the LCST1, so the copolymer $\mathrm{p}\left(\mathrm{MEO}_{2} \mathrm{MA}-\mathrm{co}-\mathrm{THPMA}\right)$ shows a hydrophilic behaviour with a coil-like conformation, which allows fluorescein molecules to be introduced into the pores of the nanocarrier. After this time, the bath temperature was increased at $37^{\circ} \mathrm{C}$ (temperature higher than the LCST1), so the copolymer shows a hydrophobic behaviour with a collapsed state, which blockes the outlets of the pores and impedes the release of fluorescein (See details in Materials and Methods section). The successful loading of cargo molecules inside the mesopores of the hybrid-MSNs was verified by the decrease in pore volume by Nitrogen adsorption porosimetry (data not shown).

Figure 3 shows the release profiles of US irradiated hybrid-MSNs and non-irradiated nanoparticles (control), in which a great difference on cargo release can be observed. At $37^{\circ} \mathrm{C}$ (T $>$ LCST1) the copolymer chains are collapsed, blocking the entrances of pores. As it has been showed above (Figure 1b), the application of ultrasounds induces the hydrolysis of THPMA groups present in the copolymer resulting onto hydrophilic methacrylic acid, MAA groups. This 
polarity change provokes the opening of the gates of the mesoporous channels resulting in a release of fluorescein to the medium. These data confirm that these hybrid-MSNs proposed can act as an effective system for US stimuli-responsive drug delivery.

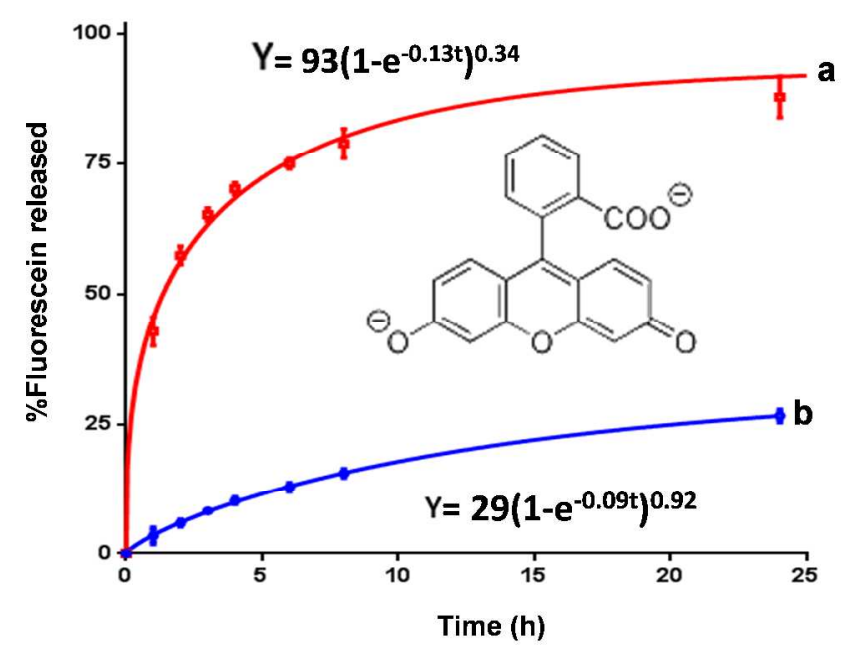

Figure 3. Release profiles of fluorescein from hybrid-MSNs in PBS solution versus time with US exposure (10 min and 1.3 MHz, $100 \mathrm{~W}$ ) (a) and without US (b).

Fluorescein release data showed in figure 3 can be fitted to a first-order kinetic model with an empirical non-ideality factor $(\delta)$ (Equation 1$){ }^{32}$

$$
\mathrm{Y}=\mathrm{A}\left(1-\mathrm{e}^{-\mathrm{kt}}\right)^{\delta}
$$

Equation 1

being $Y$ the percentage of fluorescein released at time $t, A$ the maximum amount of fluorescein released (in percentage), and $k$, the release rate constant. The values for $\delta$ are comprised between 1 for materials that follow first-order kinetics, and 0 , for materials that release the loaded drug in the very initial time of analysis. The parameters of the kinetic fitting, shown in Figure 3 and table S2, indicate that, while the maximum amount of fluorescein released is practically the totality of the loaded dye after US application, most of the cargo is retained without the stimulus. Also, the value of $\delta$ is much lower for the kinetic fitting of cargo release after ultrasound exposure $(0.34 v s$ 0.92) which is indicative of a burst release of fluorescein at very short times after the stimulus is 
applied. That is, the system shows a fast response to the external stimulus, promptly releasing its cargo.

To assure that the cargo release after US exposure is due to the mechanism above proposed and not merely because some mechanical/thermal effects caused by the US waves, similar release experiments were carried out with MSNs functionalised only with the temperature responsive polymer $\left(\mathrm{MEO}_{2} \mathrm{MA}\right)$. Theoretically, that polymer should not be sensitive to US, so if there is any cargo release that should had to be caused by some mechanical or thermal effects from the US radiation. Certainly, most of the cargo in the $\mathrm{MEO}_{2} \mathrm{MA}-\mathrm{MSNs}$ was retained after US radiation, since similar release patterns are observed with and without US stimulus (Figure S5), which validates the release mechanism above proposed. Additionally, when those studies were carried out with MSNs with no polymeric nanogates (Figure S5), the release patterns were very similar to those of US-irradiated hybrid-MSNs (Figure 3), which confirms that the latter is behaving with totally opened gates.

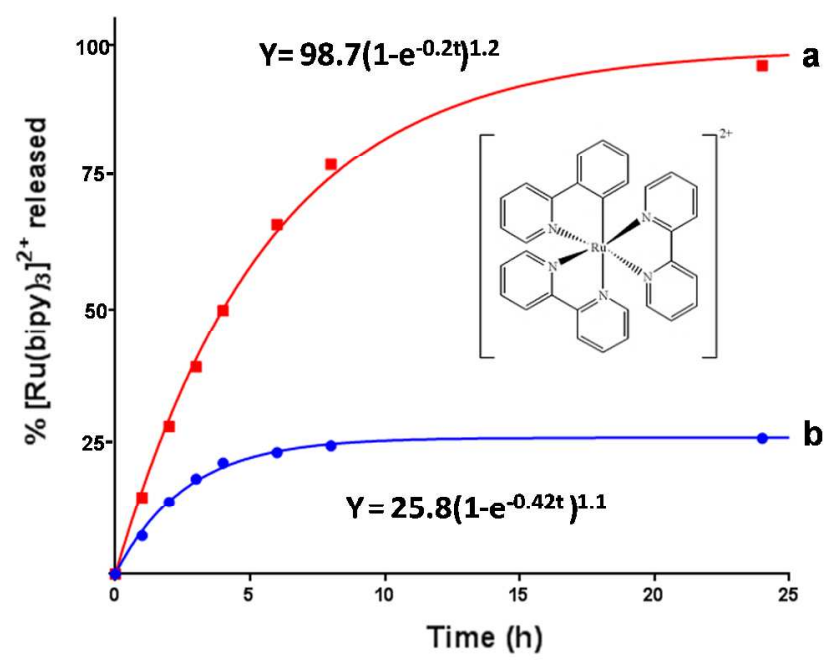

Figure 4. Release profiles of $\left[\mathrm{Ru}(\text { bipy })_{3}\right]^{2+}$ from hybrid-MSNs in PBS solution versus time with US exposure (10 min and $1.3 \mathrm{MHz}, 100 \mathrm{~W}$ ) (a) and without US (b). 
When developing stimuli-responsive nanocarriers, it is very important that they could efficiently respond to the stimuli independently of the type of cargo molecules transported. The versatility of our nanocarrier system was evaluated using a fluorescent ruthenium complex $\left[\mathrm{Ru}(\mathrm{bipy})_{3}\right]^{2+}$, which was loaded and released from the $\mathrm{p}\left(\mathrm{MEO}_{2} \mathrm{MA}-\mathrm{co}-\mathrm{THPMA}\right)$-grafted MSNs. Figure 4 shows the release patterns with and without US irradiation, which are in total agreement with those obtained with fluorescein; a good response of the material to the external stimuli, with higher amount of dye released upon US exposure compared to the amount released without stimulus. However, a tiny difference might be found in both release patterns, with a slightly slower release in the case of ruthenium complex. The negatively-charged surface of the silica walls might interact with the positively charged complex, slowing down the release. This would be in agreement with the smaller burst effect estimated in the $\delta$ value, close to 1 , in the kinetic fitting of ruthenium complex release, which means that the pore wall-cargo molecule interaction is slowing down the release kinetics. However, the difference between the release from the control (no US) and the US-irradiated nanoparticles is still very significative (Figure 4), which confirms that our versatile responsive system can work efficiently independently of the type of cargo transported.

In vitro biological evaluation. Finally, to evaluate the biological compatibility of our hybridMSNs system, nanoparticles were incubated with tumor cells (LNCaP cells, from human prostate adenocarcinoma) for two hours. The media were changed with fresh media and the cells were incubated overnight before measuring cell viability. In order to check that by-products from US exposure (like THP-ol) were not cytotoxic either, hybrid-MSNs that had been exposed to $10 \mathrm{~min}$ US irradiation (1.3 MHz and $100 \mathrm{~W})$ were also cultured with LNCaP cells under the same 
conditions. The hybrid-MSNs (with or without exposure to ultrasound) induced no toxicity at least up to $500 \mu \mathrm{g} / \mathrm{mL}$ in LNCaP cells as measured by MTS reduction assay (Figure 5).

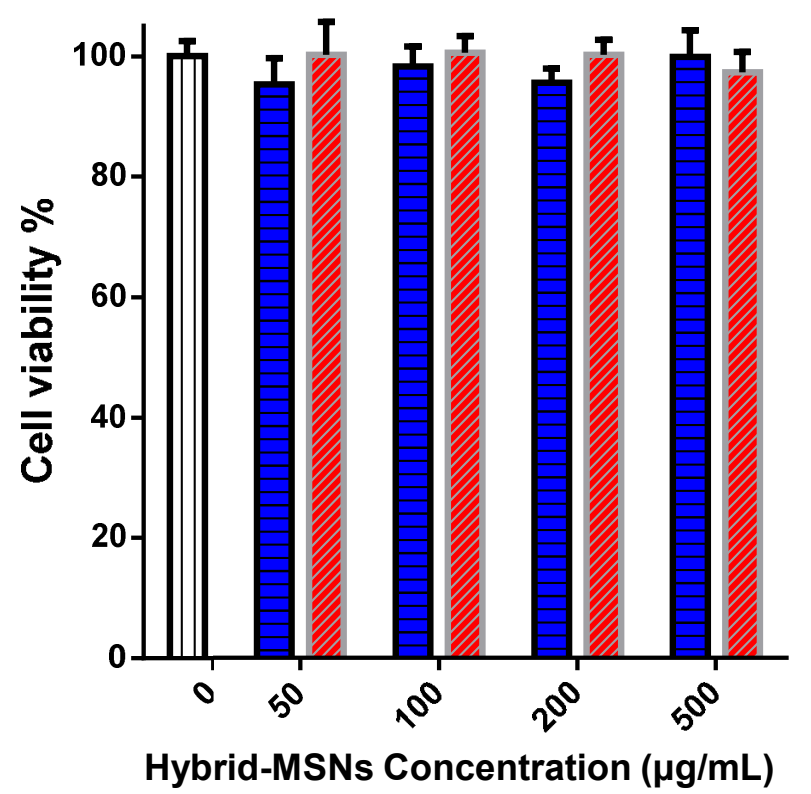

Figure 5. Cytotoxicity assay measured by MTS reduction in LNCaP cells with different concentrations of hybrid-MSNs (blue) without US exposure and (red) with US exposure (10 min and $1.3 \mathrm{MHz}, 100 \mathrm{~W}$ ).

Regarding the future application in living organisms of our hybrid system, a different US set up was investigated. In this sense, in vitro experiments were performed using a commercial US apparatus frequently used for physical therapy in humans, working at $1 \mathrm{MHz}$ and $15 \mathrm{~W}$. First, we checked the displacement of the LCST of the $\mathrm{p}\left(\mathrm{MEO}_{2} \mathrm{MA}-\mathrm{co}-\mathrm{THPMA}\right)$ after 5 min US irradiation using this equipment (Figure S6a). Also, we proved that under these US conditions no damage was produced in relevant biological molecules. Using catalase as a protein model, we did not find any change in the enzymatic activity after US application under the same conditions (Catalase enzymatic activity was determined as described elsewhere ${ }^{12}$ ) (Figure S6b). After this test, the release of a cargo after internalization of the hybrid-MSNs in cells was studied. Rhodamine B-labeled MSNs with fluorescein loaded into their mesopores were incubated with 
the same LNCaP tumor cells and fluorescence microscopy was performed to check the localization of green and red fluorescence (Figure 6). Before ultrasound exposure, green (from fluorescein loaded) and red (from rhodamine labelled) fluorescence match perfectly, which confirms that fluorescein is entrapped inside the polymer-grafted MSNs thanks to the closed polymer nanogates. Also, the perinuclear location of the nanoparticles seems to indicate that the hybrid-MSNs have been uptaken by the cells. After 5 min US irradiation of the cells with the equipment used in physical therapy, a significant part of fluorescein diffuses out of the material and stains the cell cytoplasm (Fluorescence Microscopy images taken 30 min after the US exposure was perfomed). Unequivocally, the green cytoplasm means that the nanogates of the pores were opened as a consequence of the US waves releasing the fluorescein loaded into the mesopores of the nanoparticles. This proves that our material retains its ultrasound-responsive capability even after being internalized in LNCaP cells.
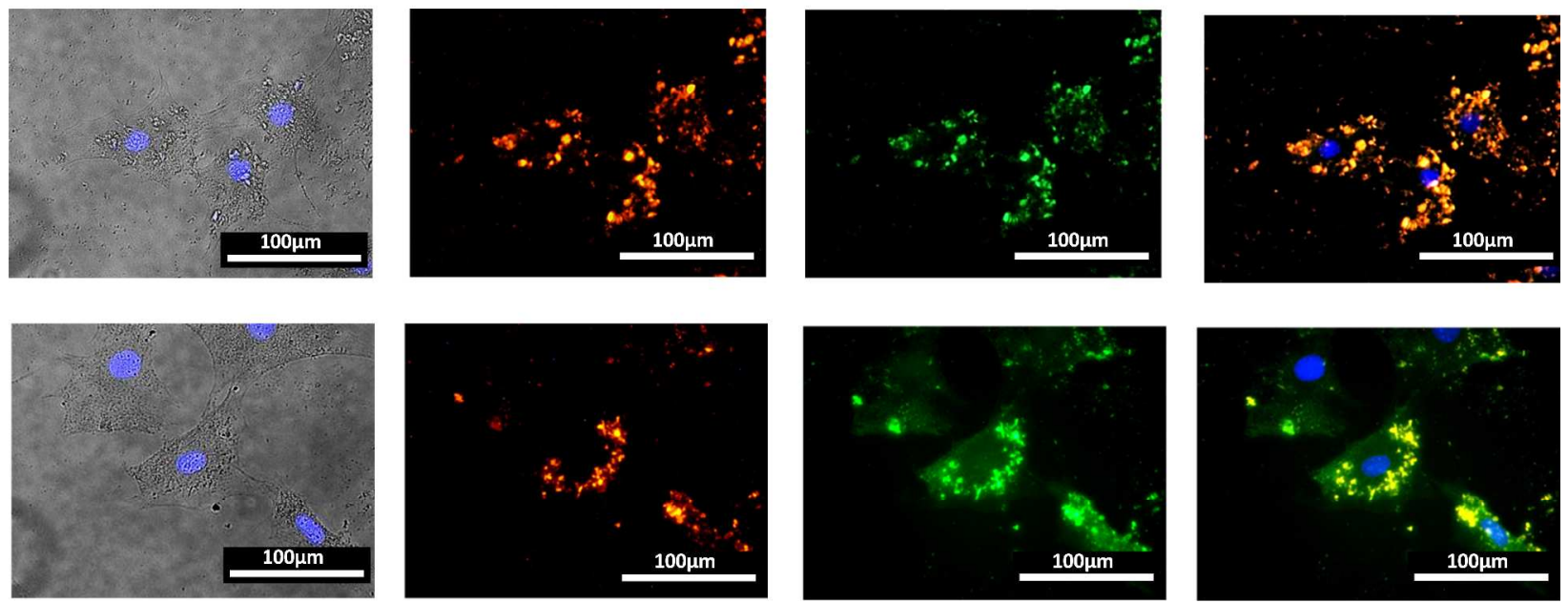

Figure 6. Fluorescence Microscopy images of LNCaP cells incubated with Rhodamine Blabeled hybrid-MSNs with fluorescein loaded before (top) and after (bottom) ultrasound irradiation. From left to right: Blue fluorescence (nuclei) and transmission images, red fluorescence (Hybrid-MSNs), green fluorescence (fluorescein cargo), overlay images of the three fluorescence channels (Fluorescence Microscopy images taken 30 min after the US exposure was perfomed). 


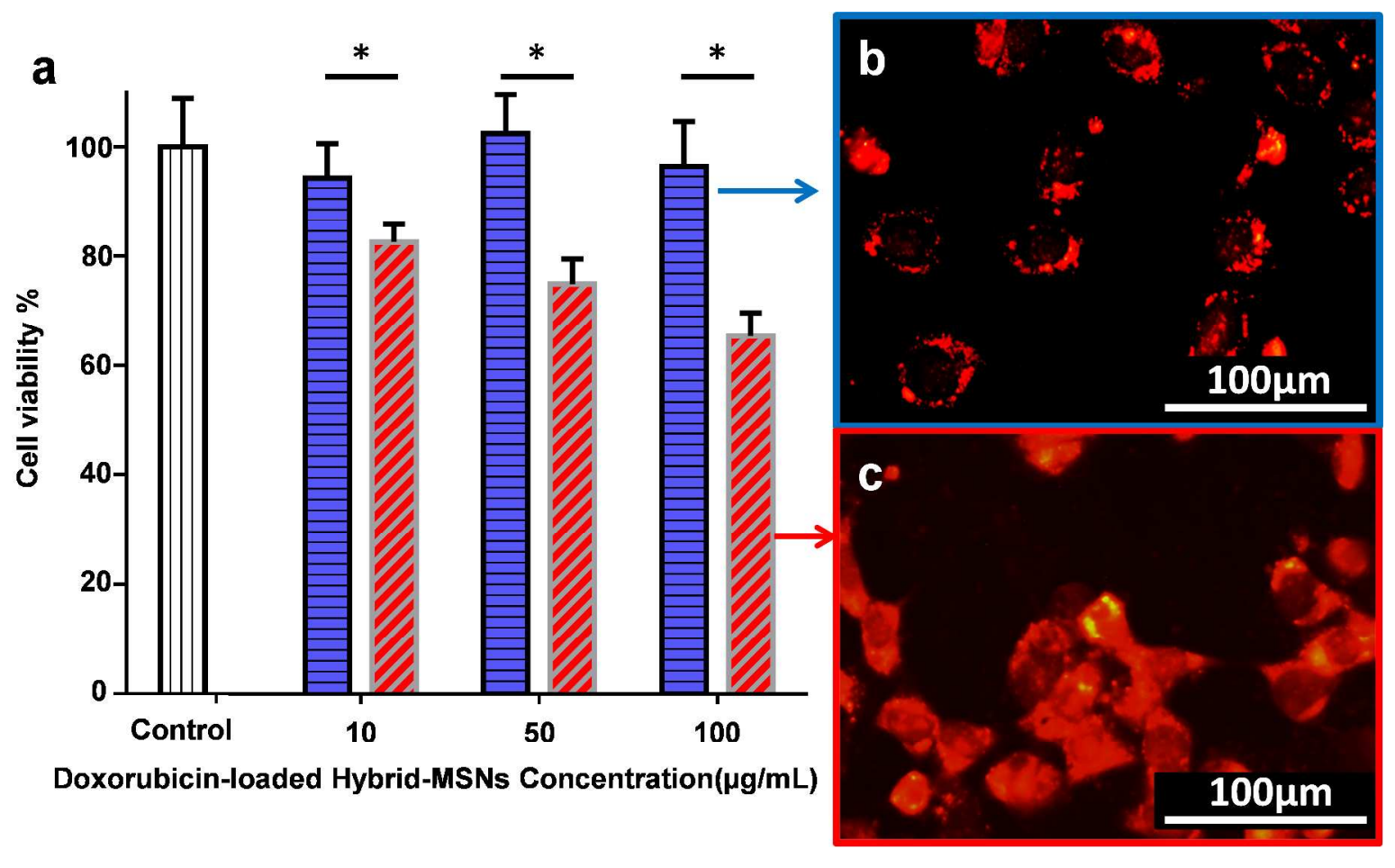

Figure 7. (a) Cytotoxicity assay measured by MTS reduction in LNCaP cells with different concentrations of doxorubicin-loaded hybrid-MSNs (blue) without US exposure and (red) with US exposure (10 min and $1.3 \mathrm{MHz}, 100 \mathrm{~W}$ ). (b) Fluorescence Microscopy images of LNCaP cells incubated with doxorubicin-loaded hybrid-MSNs (showing doxorubicin fluorescence) without US and (c) with US irradiation. (Fluorescence Microscopy images taken $24 \mathrm{~h}$ after the US exposure and nanoparticle incubation). ${ }^{*} \mathrm{p}<0.05$ (Student's $t$-test).

Finally, to test the feasibility to use this material for future anticancer therapy, doxorubicinloaded hybrid-MSNs were also cultured with LNCap cells under the same conditions as those used for the cytotoxicity study with unloaded-hybrid-MSNs (Figure 7). The results show that, while no toxicity was observed with nanoparticles not exposed to US, significant decrease in cell viability was observed at the three concentrations tested $(10,50$ and $100 \mu \mathrm{g} / \mathrm{mL})$ when the nanoparticles had been exposed to US (Figure 7a). Moreover, fluorescence microscopy images show that doxorubicin fluorescence was localized in the perinuclear region in the samples without stimulus (Figure $7 b$ ). On the other hand, doxorubicin fluorescence stained the cytoplasm of the cells with US stimulus (Figure 7c), indicating that the drug is being released from the nanoparticles only in that case. 


\section{CONCLUSIONS}

In this work, we have successfully designed and prepared a new stimuli-responsive system that uses mesoporous silica nanoparticles as carriers and polymers as nanogates sensitive to ultrasounds. The combination of the advantageous structural properties of mesoporous silica particles with an ultrasound responsive gatekeeper provides an efficient strategy for the preparation of stable and safe drug delivery system. US responsive copolymer with a LCST below $37^{\circ} \mathrm{C}$ was successfully grafted to the surface of mesoporous silica nanoparticles to close the pore entrance avoiding premature release of the loaded cargo. Upon US irradiation, the hydrophobic tetrahydropyranyl groups in the polymer backbone were cleaved leading to hydrophilic methacrylate and increasing the LCST over $37^{\circ} \mathrm{C}$. Consequently, the copolymer changed its phase state at physiological temperature and the loaded molecules were released from the nanocarriers upon US irradiation. These hybrid nanoparticles are not cytotoxic and can also be endocyted by LNCaP cells retaining their ultrasound-responsible capability because they can release the cargo inside the cells upon US irradiation. Moreover, when loaded with doxorubicin, the hybrid-mesoporous silica nanoparticles only induced cell death when they had been exposed to ultrasound.

\section{MATERIALS AND METHODS.}

Materials. Following compounds were purchased from Sigma-Aldrich Inc.: Aminopropyltriethoxysilane (APTES), ammonium nitrate, cetyltrimethylammonium bromide (CTAB), tetraethyl orthosilicate (TEOS), rhodamine B isothiocyanate (RBITC), methacrylic acid (MAA), pyridine, p-toluenesulfonic acid, toluene, dichloromethane (DCM), dihydropyran, 
dimethylformamide (DMF), 2-(2-methoxyethoxy) ethylmethacrylate ( $\left.\mathrm{MEO}_{2} \mathrm{MA}\right), 4,4$ '-Azobis(4cyanovaleric acid) (ABCVA), diethylether, N,N'-Dicyclohexylcarbodiimide (DCC), NHydroxysuccinimide (NHS), catalase, phosphate-buffered solution (PBS), fluorescein sodium salt and $\left[\mathrm{Ru}(\text { bipy })_{3}\right] \mathrm{Cl}_{2}$. These compounds were used without further purification.

Characterization Techniques. The materials were analysed by X-ray diffraction (XRD) in a Philips X-Pert MPD diffractometer equipped with $\mathrm{Cu} \mathrm{K} \alpha$ radiation. Thermogravimetry and Differential Temperature Analyses (TGA/DTA) were performed in a Perkin Elmer Pyris Diamond TG/DTA analyser, with $5^{\circ} \mathrm{C} / \mathrm{min}$ heating ramps, from room temperature to $600^{\circ} \mathrm{C}$. Fourier Transform Infrared (FTIR) spectra were obtained in a Nicolet (Thermo Fisher Scientific) Nexus spectrometer equipped with a Smart Golden Gate ATR accessory. Surface morphology was analysed by Scanning Electron Microscopy (SEM) in a JEOL 6400 Electron microscope. Transmission electron microscopy (TEM) was carried out with a JEOL JEM 2100 instrument operated at $200 \mathrm{kV}$, equipped with a CCD camera (KeenView Camera). $\mathrm{N}_{2}$ adsorption was carried out on a Micromeritics ASAP 2010 instrument; surface area was obtained by applying the BET method to the isotherm and the pore size distribution was determined by the BJH method from the desorption branch of the isotherm. The mesopore diameter was determined from the maximum of the pore size distribution curve. The zeta potential and hydrodynamic size of nanoparticles were measured by means of a Zetasizer Nano ZS (Malvern Instruments) equipped with a $633 \mathrm{~nm}$ "red" laser. ${ }^{1} \mathrm{H}$ NMR experiments were carried out in a Bruker AV $250 \mathrm{MHz}$ apparatus. To determine the molecular weight distribution of the copolymers, Gel Permeation Chromatography (GPC) was performed in a Waters Alliance automatic analysis system with a Model \#2695 separations module coupled to a Model \#2414 Refractive Index Detector. GPC measurements were carried out using polyethylene (Glycol/Oxide) standards and 
DMF with $\mathrm{LiCl} 10 \mathrm{mM}$ as the eluent. Fluorescence spectrometry was used to determine cargo release by means of a Biotek Synergy 4 device. Fluorescence microscopy was performed with an Evos ${ }^{\circledR}$ FL Cell Imaging System equipped with tree Led Lights Cubes (IEX (nm); 1EM (nm)): DAPY (357/44; 447/60), GFP (470/22; 525/50), RFP (531/40; 593/40) from AMG (Advance Microscopy Group).

Synthesis of mesoporous silica nanoparticles (MSNs). Mesoporous silica nanoparticles, MSNs, were synthesized by the modified Stöber method from TEOS in the presence of CTAB, as structure-directing agent: $1 \mathrm{~g}$ of CTAB, $480 \mathrm{~mL}$ of $\mathrm{H}_{2} \mathrm{O}$ (Milli-Q) and $3.5 \mathrm{~mL}$ of $\mathrm{NaOH}(2 \mathrm{M})$ were added to a $1000 \mathrm{~mL}$ round-bottom flask. The mixture was heated to $80{ }^{\circ} \mathrm{C}$ and stirred at $600 \mathrm{rpm}$. Then, $5 \mathrm{~mL}$ of TEOS were added dropwise at $0.25 \mathrm{~mL} / \mathrm{min}$ rate with a pump. The white suspension obtained was magnetically stirred for further $2 \mathrm{~h}$ at $80{ }^{\circ} \mathrm{C}$. Then, the reaction mixture was centrifuged and washed with water and ethanol. Finally, the surfactant was removed by ionic exchange using a solution of amonium nitrate $(10 \mathrm{mg} / \mathrm{mL})$ in ethanol $(95 \%)$ at $70{ }^{\circ} \mathrm{C}$ overnight under magnetic stirring. The nanoparticles were collected by centrifugation, washed with ethanol three times and dried under vacuum overnight.

Rhodamine B-labelled nanoparticles were synthesized by reacting $1 \mathrm{mg}$ of Rhodamine-B isothiocyanate with $2.2 \mu \mathrm{L}$ APTES in $100 \mu \mathrm{L}$ ethanol for $2 \mathrm{~h}$. Then the reaction mixture was added with the $5 \mathrm{~mL}$ of TEOS as previously described.

\section{Synthesis and characterization of ultrasound-responsive monomer, tetrahydropyranyl}

methacrylate (THPMA). THPMA was synthesized following a previously described method. ${ }^{33}$ Briefly, methacrylic acid $(8 \mathrm{~g})$, pyridine $(0.3 \mathrm{~mL})$, and p-toluenesulfonic acid $(0.7 \mathrm{~g})$ were dissolved in $80 \mathrm{~mL}$ of dichloromethane (Figure S7a). Dihydropyran (0.162 mol) was slowly added at room temperature. After stirring overnight, the solution was filtered by a short silica 
column. The solution was extracted by water and brine three times. Finally, the solvent was removed under vacuum to yield THPMA. The obtained product was characterized by ${ }^{1} \mathrm{H}$ NMR (Figure S7b).

\section{Synthesis and characterization of the temperature and ultrasound-responsive copolymer} p(MEO 2 MA-co-THPMA). The copolymer, poly(2-(2methoxyethoxy)ethylmethacrylate-co-2tetrahydropyranyl methacrylate), $\mathrm{p}\left(\mathrm{MEO}_{2} \mathrm{MA}-\mathrm{co}-\mathrm{THPMA}\right)$, was synthesized by free radical polymerization from $\mathrm{MEO}_{2} \mathrm{MA}$ (temperature-responsive monomer) and THPMA (ultrasoundresponsive monomer) (Figure S8a). Briefly, $\mathrm{MEO}_{2} \mathrm{MA}$ and THPMA at different molar ratios (0.01 mol in total) were placed in a seal vial and purged with nitrogen. $16 \mathrm{~mL}$ of DMF were added under inert atmosphere and the solution was placed at $80^{\circ} \mathrm{C}$ under magnetic stirring. $1 \mathrm{~mL}$ DMF with 0.003 mmol of initiator (ABCVA) was added. The reaction was carried out overnight. Then, the polymer was precipitated in cold diethylether, separated by centrifugation and washed 3 times with diethylether followed by evaporation of the solvent. The polymer was characterized by ${ }^{1}$ H NMR (Figure S8b), FTIR spectroscopy (Figure S3) and GPC. The obtained copolymer has a number-average molecular weight, determined by GPC, of 27094 Da with a polydispersity of 1.83 .

In order to check the changes in the polymer structure after US exposure, the aqueous solutions of the polymers after the application of the stimulus were freeze-dried. Then, the polymers were washed with diethyl ether and, after the evaporation of the ether, they were dissolved in $\mathrm{CDCl}_{3}$ and ${ }^{1} \mathrm{H}$ NMR spectra were acquired.

Preparation of polymer grafted MSN nanoparticles. $0.3 \mathrm{~g}$ of carboxylic acid-terminated poly(MEO $\left.{ }_{2} \mathrm{MA}-\mathrm{co}-\mathrm{THPMA}\right), 11 \mathrm{mg} \mathrm{DCC}$ and $6 \mathrm{mg}$ NHS were added to a glass vial. The vial was purged with nitrogen and $2 \mathrm{~mL}$ DMF were added. Then, under $\mathrm{N}_{2}$ atmosphere and with 
magnetic stirring, DMF (1 mL) with $8 \mu$ APTES were added. The solution was stirred overnight (Solution 1, sililated copolymer solution). Then, $1 \mathrm{~mL}$ of Solution 1 was added dropwise to 20 $\mathrm{mL}$ of toluene containing $50 \mathrm{mg}$ of MSNs under vigorous stirring. The reaction medium was heated under reflux. After $4 \mathrm{~h}$, another $\mathrm{mL}$ of Solution 1 was added. $4 \mathrm{~h}$ later, the remaining Solution 1 was added. The reaction was left under vigorous stirring for $24 \mathrm{~h}$. Then, the hybrid MSNs were collected by centrifugation and washed with toluene, DMF (twice), cold water (twice) and ethanol. Afterwards, the nanoparticles were dried under vacuum for $16 \mathrm{~h}$ (Scheme 2).

Determination of Phase Transition Temperatures (LCST). The LCST was determined by Dynamic Light Scattering (DLS) by means of the drastic change in the scattering intensity obtained by the precipitation of the polymer at the LCST (determined as the temperature at which the scattering intensity is $50 \%$ of the maximum). Measurement of the LCST was performed using a Zetasizer Nano ZS (Malvern Instruments) equipped with a 633 nm "red" laser. To determine the transition temperature, the temperature dependence of the scattering intensity at $90^{\circ}$ from $1 \mathrm{~mL}$ of solution in a glass cuvette was measured. The temperature was increased by discrete temperature increments in the range $10-45^{\circ} \mathrm{C}$, and the readings were taken after 2 min equilibration at each temperature.

Nanoparticles degradation studies. In order to study nanoparticle degradation in PBS, several aliquots of $1 \mathrm{mg}$ of MSNs or hybrid-MSNs were dispersed in $1 \mathrm{ml}$ of PBS each (10mM $\mathrm{pH}=7.4$ ) in Eppendorf tubes. Every $24 \mathrm{~h}$, the nanoparticles were centrifuged and redispersed in fresh PBS. At 5, 8 and 12 days, some of the samples were washed with deionized water and observed by Transmission Electron Microscopy.

Cargo loading and release. Cargo loading: $20 \mathrm{mg}$ of nanoparticles were placed in a glass vial with a septum and dried at $80^{\circ} \mathrm{C}$ under vacuum for $24 \mathrm{~h}$. Then, the vial was placed at $4^{\circ} \mathrm{C}$ 
with magnetic stirring and $5 \mathrm{~mL}$ of cargo solution $\left(20 \mathrm{mg} / \mathrm{mL}\right.$, fluorescein or $\mathrm{Ru}(\text { bipy })_{3}{ }^{2+}$ in PBS) were added and the suspension was stirred at $4^{\circ} \mathrm{C}$ for $24 \mathrm{~h}$. After that time, the sample was filtered and washed two times with previously hot $\mathrm{PBS}\left(50^{\circ} \mathrm{C}\right)$ in order to remove the cargo absorbed on the external surface. Finally, the products were dried under vacuum at $25^{\circ} \mathrm{C}$.

Cargo Release: $9 \mathrm{mg}$ of cargo-loaded nanoparticles were suspended in $1.8 \mathrm{~mL}$ of fresh PBS pH $7.4(10 \mathrm{mM})$. Then, $0.5 \mathrm{~mL}$ of nanoparticles suspension were placed on a Transwell permeable support with $0.4 \mu \mathrm{m}$ of polycarbonate membrane (3 replicas were performed). The well was filled with $1.5 \mathrm{~mL}$ of PBS $\mathrm{pH} 7.4(10 \mathrm{mM})$ and the suspension was stirred at $37^{\circ} \mathrm{C}$ and $100 \mathrm{rpm}$ during all the experiment. To study the US responsiveness of the cargo-loaded nanoparticles, the particles suspension was subjected to US exposure (10 min at $1.3 \mathrm{MHz}$ and $100 \mathrm{~W}$ ) before to place the $0.5 \mathrm{~mL}$ on a Transwell. At every time point studied, the solution outside the transwell insert was replaced with fresh medium and the amount of cargo released was determined by fluorescence spectrometry (Fluorescein: $\lambda \operatorname{exc} 490, \lambda \mathrm{em} 514 \mathrm{~nm}$; Ru(bipy) ${ }^{2+}$ : $\lambda \operatorname{exc} 454, \lambda \operatorname{em} 593 \mathrm{~nm})$.

Cytotoxicity assays and intracelular fluorescein release. LNCaP cells were plated $24 \mathrm{~h}$ before the start of the experiment in 12 well plates at a density of $10^{4}$ cells per $\mathrm{cm}^{2}$. The cytotoxicity of hybrid-MSNs was evaluated using the standard MTS assay protocol using a commercial assay and following the manufacturer protocol (CellTiter ${ }^{\circ}$ Aqueous One Solution Cell Proliferation Assay). Briefly, LNCaP cells were incubated with various concentrations of hybrid-MSNs for $2 \mathrm{~h}(\mathrm{n}=3)$. To study the toxicity induced by US-exposed hybrid-MSNs, the nanoparticles were exposed to ultrasound as described for the in vial cargo released studies before incubating them with the cells. The cells were then cultured for another $24 \mathrm{~h}$. The medium was then replaced with $600 \mu \mathrm{L}$ culture medium including MTS, and the incubation proceeded for 
$3 \mathrm{~h}$. The medium was then removed, and its absorption at $490 \mathrm{~nm}$ was measured using a microplate reader. The same procedure was used to determine the cytotoxicity of doxorubicinloaded MSNs. In this case, before measuring cell viability, the culture media was replaced with fresh media and fluorescence microscopy images were taken to determine the location of doxorubicin in the cells.

For the in vitro intracellular release experiments, rhodamine B-labeled hybrid-MSNs with fluorescein loaded were used. LNCaP cells were incubated with the nanoparticles for $2 \mathrm{~h}$ in serum-free culture medium. Then, the medium was withdrawn and cells were washed with PBS three times. Cells were fixed with isopropanol for $2 \mathrm{~min}$ and stained with DAPI. Fluorescence microscopy images were taken to evaluate cargo localization before and after US exposure (5 min at $1 \mathrm{MHz}$ and $15 \mathrm{~W}$ ). Red channel was used to locate nanoparticles, green for cargo and blue for cell nucleus.

Ultrasonic experiments. All the US experiments, except those related with the intracellular release studies, were performed in a commercial ultrasound apparatus, which generates an ultrasound beam of adjustable power (up to $100 \mathrm{~W}$ ) at a frequency of $1.3 \mathrm{MHz}$. In the experiments, a power of $100 \mathrm{~W}$ was used. The tube reactor, a cylindrical glass vial $(4.5 \mathrm{x} 1 \mathrm{~cm})$ with a latex cover, containing $1.5 \mathrm{~mL}$ of the polymer solution $(5 \mathrm{mg} / \mathrm{mL})$ or $1.8 \mathrm{~mL}$ of nanoparticles suspension $(5 \mathrm{mg} / \mathrm{mL})$ was immersed in a water tank (Figure S9a). The tube was positioned at the centre of the ultrasound beam and at $3 \mathrm{~cm}$ of the acoustic lens transducer. The US beam can penetrate through the latex cover and act on the solution placed in the tube reactor. After the ultrasound irradiation, the tube reactor was removed from the tank and used.

In order to study if the US beam can penetrate trough the cells ant acts over loaded nanoparticles releasing the cargo, in vitro intracellular cargo release experiments, a commercial 
ultrasound apparatus for application in physical therapy was used (Pagani Sonoquartz Evo). The parameters selected were: $1 \mathrm{MHz}, 15 \mathrm{~W}$, continuous application, 5min. Ultrasounds were applied from the top of a filled culture well through a latex membrane (ultrasound transmission gel was placed between the transducer and the latex membrane) (Figure S9b).

Conflict of Interest: The authors declare no competing financial interest.

Acknowledgments. Financial support from Ministerio de Economía y Competitividad, Spain (Project MAT2012-35556 and Project CSO2010-11384-E, Ageing Network of Excellence) and CIBER-BBN are gratefully acknowledged. CIBER-BBN is an initiative funded by the VI National R\&D\&i Plan 2008-2011, Iniciativa Ingenio 2010, Consolider Program, CIBER Actions and financed by the Instituto de Salud Carlos III with assistance from the European Regional Development Fund. The XRD measurements and ${ }^{1} \mathrm{H}-\mathrm{NMR}$ spectra were performed at C.A.I Difracción de Rayos X and Resonancia Magnética Nuclear, UCM (Spain), respectively. SEM and TEM were performed at ICTS National Centre for Electron Microscopy (Spain). JL Paris gratefully acknowledges Ministerio de Economía y Competitividad, Spain, for his PhD grant (BES-2013-064182).

Supporting Information Available. Temperature responsive monomer: US responsive monomer $\left(\mathrm{MEO}_{2} \mathrm{MA}\right.$ :THPMA) ratios tested for the dual $\mathrm{p}\left(\mathrm{MEO}_{2} \mathrm{MA}-\mathrm{co}-\mathrm{THPMA}\right)$ synthesis (table S1), experimental conditions for the copolymer grafting to silica nanoparticles (table S2) and cargo release kinetic parameters (table S3). LSCT and ${ }^{1} \mathrm{H}$ NMR spectra of the $\mathrm{p}\left(\mathrm{MEO}_{2} \mathrm{MA}\right)$ before and after US (Figure S1), ${ }^{1} \mathrm{H}$ NMR spectra of $\mathrm{p}\left(\mathrm{MEO}_{2} \mathrm{MA}-\mathrm{co}-\mathrm{THPMA}\right)$ with different treatments and LCST before and after heating at $80^{\circ} \mathrm{C}$ for $30 \mathrm{~min}$ (Figure S2), XRD, FTIR TGA and Nitrogen adsorption/desorption corresponding to MSNs and hybrid-MSNs (Figure S3), TEM micrographs of MSNs and hybrid-MSNs suspended in PBS for different periods of time (Figure 
S4), release experiments corresponding to MSNs and $\mathrm{p}\left(\mathrm{MEO}_{2} \mathrm{MA}\right)-\mathrm{MSNs}$ (Figure S5), LCST of $\mathrm{p}\left(\mathrm{MEO}_{2} \mathrm{MA}-\mathrm{co}-\mathrm{THPMA}\right)$ and Catalase enzymatic activity before and after US exposure with a US physical therapy apparatus (Figure S6), synthesis scheme and ${ }^{1} \mathrm{H}-\mathrm{NMR}$ characterization of ultrasound monomer (THPMA) (Figure S7) and copolymer poly( $\mathrm{MEO}_{2} \mathrm{MA}$-co-THPMA) (Figure S8), schematic representation of the two ultrasound systems used in this work (Figure S9). This material is available free of charge via the Internet at http://pubs.acs.org.

\section{AUTHOR INFORMATION}

\section{Corresponding Author}

*María Vallet-Regí. Dpto. Química Inorgánica y Bioinorgánica, Facultad de Farmacia, UCM, 28040-Madrid, Spain. e-mail: vallet@ucm.es

\section{Author Contributions}

The manuscript was written through contributions of all authors. All authors have given approval to the final version of the manuscript.

\section{REFERENCES AND NOTES}

(1) Farokhzad, O. C.; Langer, R. Impact of Nanotechnology on Drug \nDelivery. ACS Nano 2009, 3, 16-20.

(2) Steichen, S. D.; Caldorera-Moore, M.; Peppas, N. a. A Review of Current Nanoparticle and Targeting Moieties for the Delivery of Cancer Therapeutics. Eur. J. Pharm. Sci. 2013, 48, 416-427.

(3) Bogart, L. K.; Pourroy, G.; Murphy, C. J.; Puntes, V.; Pellegrino, T.; Rosenblum, D.; Peer, D.; Lévy, R. Nanoparticles for Imaging, Sensing, and Therapeutic Intervention. ACS Nano 2014, 8, 3107-3122.

(4) Manzano, M.; Vallet-Regí, M. New Developments in Ordered Mesoporous Materials for Drug Delivery. J. Mater. Chem. 2010, 20, 5593.

(5) Vivero-Escoto, J. L.; Slowing, I. I.; Trewyn, B. G.; Lin, V. S.-Y. Mesoporous Silica Nanoparticles for Intracellular Controlled Drug Delivery. Small 2010, 6, 1952-1967.

(6) Li, Z.; Barnes, J. C.; Bosoy, A.; Stoddart, J. F.; Zink, J. I. Mesoporous Silica Nanoparticles in Biomedical Applications. Chem. Soc. Rev. 2012, 41, 2590. 
(7) Baeza, A.; Colilla, M.; Vallet-Regí, M. Advances in Mesoporous Silica Nanoparticles for Targeted Stimuli-Responsive Drug Delivery. Expert Opin. Drug Deliv. 2015, 12, 319337.

(8) Vallet-Regi, M.; Rámila, a.; Del Real, R. P.; Pérez-Pariente, J. A New Property of MCM41: Drug Delivery System. Chem. Mater. 2001, 13, 308-311.

(9) Tarn, D.; Ashley, C. E.; Xue, M.; Carnes, E. C.; Zink, J. I.; Brinker, C. J. Mesoporous Silica Nanoparticle Nanocarriers: Biofunctionality and Biocompatibility. Acc. Chem. Res. 2013, 46, 792-801.

(10) Argyo, C.; Weiss, V.; Bräuchle, C.; Bein, T. Multifunctional Mesoporous Silica Nanoparticles as a Universal Platform for Drug Delivery. Chem. Mater. 2014, 26, 435451.

(11) Mura, S.; Nicolas, J.; Couvreur, P. Stimuli-Responsive Nanocarriers for Drug Delivery. Nat. Mater. 2013, 12, 991-1003.

(12) Baeza, A.; Guisasola, E.; Ruiz-Hernández, E.; Vallet-Regí, M. Magnetically Triggered Multidrug Release by Hybrid Mesoporous Silica Nanoparticles. Chem. Mater. 2012, 24, 517-524.

(13) Lai, J.; Mu, X.; Xu, Y.; Wu, X.; Wu, C.; Li, C.; Chen, J.; Zhao, Y. Light-Responsive Nanogated Ensemble Based on Polymer Grafted Mesoporous Silica Hybrid Nanoparticles. Chem. Commun. (Camb). 2010, 46, 7370-7372.

(14) Martínez-Carmona, M.; Baeza, A.; Rodriguez-Milla, M. A.; García-Castro, J.; ValletRegí, M. Mesoporous Silica Nanoparticles Grafted with a Light-Responsive Protein Shell for Highly Cytotoxic Antitumoral Therapy. J. Mater. Chem. B 2015, 3, 5746-5752.

(15) Ruiz-Hernández, E.; Baeza, A.; Vallet-Regí, M. Smart Drug Delivery through DNA/magnetic Nanoparticle Gates. ACS Nano 2011, 5, 1259-1266.

(16) Sirsi, S. R.; Borden, M. A. State-of-the-Art Materials for Ultrasound-Triggered Drug Delivery. Adv. Drug Deliv. Rev. 2014, 72, 3-14.

(17) Rapoport, N.; Nam, K. H.; Gupta, R.; Gao, Z.; Mohan, P.; Payne, A.; Todd, N.; Liu, X.; Kim, T.; Shea, J.; et al. Ultrasound-Mediated Tumor Imaging and Nanotherapy Using Drug Loaded, Block Copolymer Stabilized Perfluorocarbon Nanoemulsions. J. Control. Release 2011, 153, 4-15.

(18) Wood, A. K. W.; Sehgal, C. M. A Review of Low-Intensity Ultrasound for Cancer Therapy. Ultrasound Med. Biol. 2015, 41, 905-928.

(19) Cintas, P.; Tagliapietra, S.; Caporaso, M.; Tabasso, S.; Cravotto, G. Ultrasonics Sonochemistry Enabling Technologies Built on a Sonochemical Platform : Challenges and Opportunities. Ultrason. - Sonochemistry 2015, 25, 8-16.

(20) Wang, J.; Pelletier, M.; Zhang, H.; Xia, H.; Zhao, Y. High-Frequency UltrasoundResponsive Block Copolymer Micelle. Langmuir 2009, 25, 13201-13205.

(21) Brantley, J. N.; Wiggins, K. M.; Bielawski, C. W. Unclicking the Click: Mechanically Facilitated 1,3-Dipolar Cycloreversions. Science 2011, 333, 1606-1609.

(22) Xuan, J.; Boissière, O.; Zhao, Y.; Yan, B.; Tremblay, L.; Lacelle, S.; Xia, H.; Zhao, Y. Ultrasound-Responsive Block Copolymer Micelles Based on a New Amplification Mechanism. Langmuir 2012, 28, 16463-16468.

(23) You, Y. Z.; Kalebaila, K. K.; Brock, S. L.; Oupický, D. Temperature-Controlled Uptake and Release in PNIPAM-Modified Porous Silica Nanoparticles. Chem. Mater. 2008, 20, 3354-3359. 
(24) Chen, J.; Liu, M.; Chen, C.; Gong, H.; Gao, C. Synthesis and Characterization of Silica Nanoparticles with Well-Defined Thermoresponsive PNIPAM via a Combination of RAFT and Click Chemistry. ACS Appl. Mater. Interfaces 2011, 3, 3215-3223.

(25) Liu, R.; Zhang, Y.; Zhao, X.; Agarwal, A.; Mueller, L. J.; Feng, P. pH-Responsive Nanogated Ensemble Based on Gold-Capped Mesoporous Silica through an Acid-Labile Acetal Linker. J. Am. Chem. Soc. 2010, 132, 1500-1501.

(26) Lutz, J.-F. J.-F.; Hoth, A.; Schade, K. Design of Oligo(ethylene \{glycol)-Based\} Thermoresponsive Polymers: An Optimization Study. Des. Monomers Polym. 2009, 12, $343-353$.

(27) Jung, J.; Lee, I.-H.; Lee, E.; Park, J.; Jon, S. pH-Sensitive Polymer Nanospheres for Use as a Potential Drug Delivery Vehicle. Biomacromolecules 2007, 8, 3401-3407.

(28) Lutz, J. F. Polymerization of Oligo(ethylene Glycol) (meth)acrylates: Toward New Generations of Smart Biocompatible Materials. J. Polym. Sci. Part A Polym. Chem. 2008, 46, 3459-3470.

(29) Kim, H. J.; Matsuda, H.; Zhou, H.; Honma, I. Ultrasound-Triggered Smart Drug Release from a Poly(dimethylsiloxane)- Mesoporous Silica Composite. Adv. Mater. 2006, 18, 3083-3088.

(30) Kwon, E. J.; Lee, T. G. Surface-Modified Mesoporous Silica with Ferrocene Derivatives and Its Ultrasound-Triggered Functionality. Appl. Surf. Sci. 2008, 254, 4732-4737.

(31) Cauda, V.; Schlossbauer, A.; Bein, T. Bio-Degradation Study of Colloidal Mesoporous Silica Nanoparticles: Effect of Surface Functionalization with Organo-Silanes and Poly(ethylene Glycol). Microporous Mesoporous Mater. 2010, 132, 60-71.

(32) Nieto, A.; Balas, F.; Colilla, M.; Manzano, M.; Vallet-Regí, M. Functionalization Degree of SBA-15 as Key Factor to Modulate Sodium Alendronate Dosage. Microporous Mesoporous Mater. 2008, 116, 4-13.

(33) Lee, J.-T.; George, M. C.; Moore, J. S.; Braun, P. V. Multiphoton Writing of ThreeDimensional Fluidic Channels within a Porous Matrix. J. Am. Chem. Soc. 2009, 131, 11294-11295. 
Insert Table of Contents Graphic and Synopsis Here

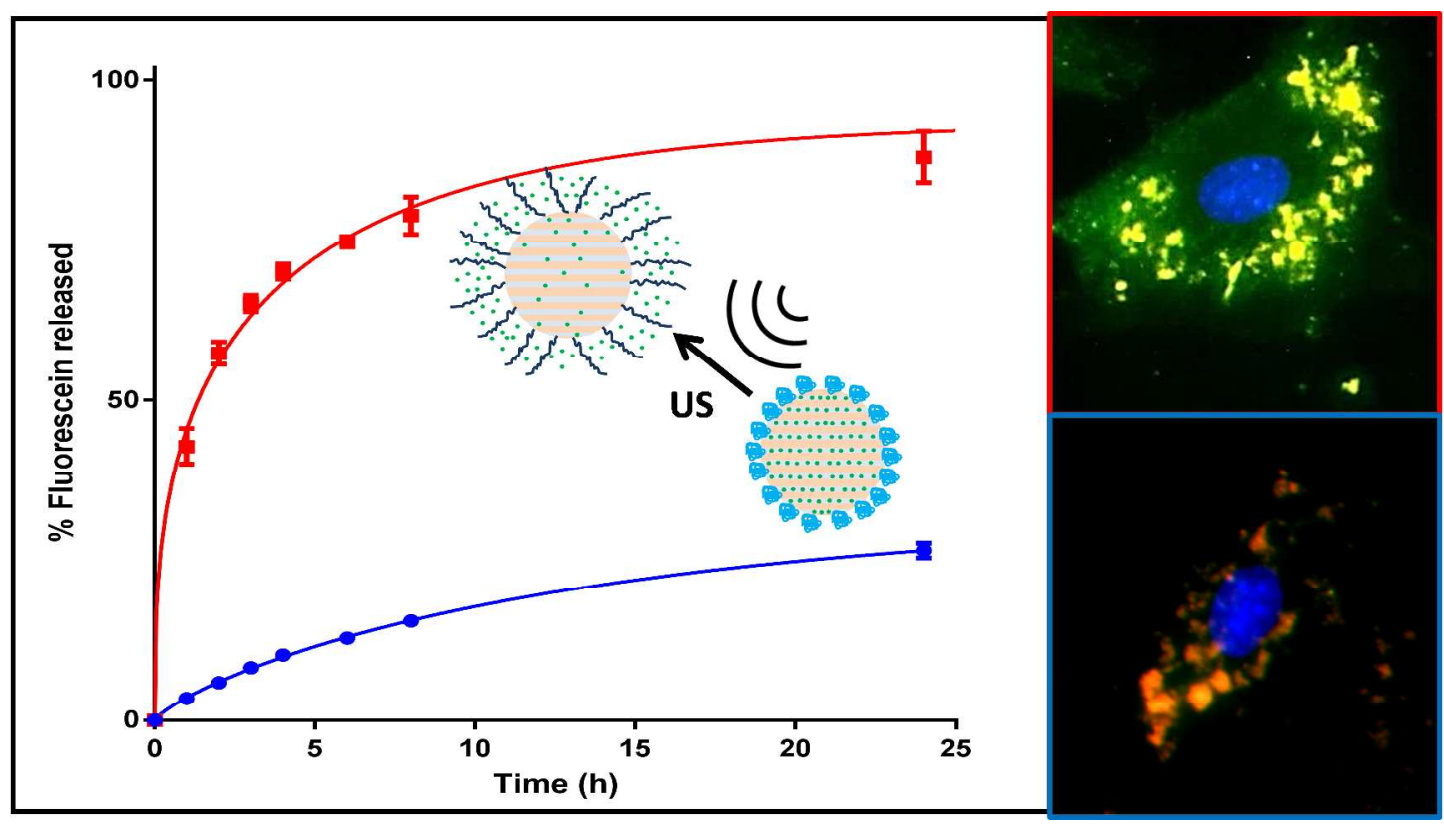

